\section{$\mathbb{T}$ Periodica Polytechnica Civil Engineering}

\author{
62(1), pp. 267-276, 2018 \\ https://doi.org/10.3311/PPci.11551 \\ Creative Commons Attribution (i)
}

\title{
Parametric Study on the Element Size Effect for Optimal Topologies
}

\author{
Piotr Tauzowski ${ }^{1}$, János Lógó ${ }^{*}$, Erika Pintér ${ }^{2}$
}

Received 12 December 2016; Revised 10 August 2017; Accepted 14 August 2017

\begin{abstract}
Topology optimization is complex engineering design tool. It needs intensive mathematical, mechanical and computing tools to perform the required design. During its hundred years of history it has become clear that the non-unique solution property of the method is affected by the material parameters (Poisson ratio) and the ways of the discretization. The aim of the paper is to investigate the influence of parameter changes to optimal design property in tasks with great number of degrees of freedom. The parametric study includes influence of material parameter (Poisson ratio) as well as the size of the ground elements which are commonly applied during the discretization. Increasing the size of the ground elements while the total number of the finite elements is constant, the computational time is significantly reduced. Therefore the study on changing accuracy versus ground element resolution may be important factor in choosing ground element size. In addition to it the effective properties of arrangements of the strong and weak materials (black and white elements) in a checkerboard fashion are also investigated. The Michell-type problem is investigated by the minimization of the weight of the structure subjected to a compliance constraint.
\end{abstract}

\section{Keywords}

topology optimization, element size, singular topologies, checker board pattern, ground element

\footnotetext{
1 Institute of Fundamental Technological Research, Polish Academy of Sciences,

P1-02-106 Warszawa, Pawińskiego 5B, Poland

2 Department of Structural Mechanics

Faculty of Civil Engineering,

Budapest University of Technology and Economics

H-1521 Budapest, P.O.B. 91, Hungary

* Corresponding author's email: logo@ep-mech.me.bme.hu
}

\section{Introduction}

The engineering design is a very complex work. The designers have to take into consideration external (loading, design domain) and internal (effect of the numerical approximations) uncertain data and effect during this procedure. Sometimes the initial loading information has to recalculate (optimize) before the design [20] or due to the multiple solutions the designers have to select the most appropriate one. In engineering one can find an effective tool for these questions in topology optimization [15]. Topology optimization is one of the most popular parts of structural optimization. The "modern" period has been counted since the seminal paper of Bendsoe and Kikuchi in 1988 [4]. Topology optimization is a complex engineering design tool. It needs intensive mathematical, mechanical and computing tools to perform the required design. The method and the different solution techniques can be followed in several publications $[1-3,5,7,10,15,17,19]$. It has reached a rather high level of reputation in almost all field of life including many industrial fields and it has widespread academic use for structural optimization problems and also for material, mechanism, electromagnetics and other coupled field of design. Despite the level of research in topology optimization, several problems still exist concerning convergence, checkerboards and mesh-dependence which are subject to debate in the topology optimization community $[6,8,9$, 13-16]. During its hundred years of history it has become clear that the non-unique solution property of the method is affected by the material parameters (Poisson ratio) and the ways of the discretization. The applied finite element technique and the selected type of finite elements (generally four-nodes quadrilateral elements are used) can overcome numerical difficulties [9, 10-11, 18-19]. From the very first start of the numerical solution technique of topology optimization, a serious problem with it was the erroneous appearance of corner contacts between solid elements in the solution (checkerboards, diagonal element chains, isolated hinges). To overcome this problem different techniques (some of them are heuristic) were applied $[6,8-10,13-16,18]$. 
The aim of the paper is to investigate the influence of parameter changes to optimal design property in a task with great number of degrees of freedom. The parametric study includes influence of material parameter $v$ (Poisson ratio) as well as the size of the ground element which is commonly applied during the discretization. Increasing the size of the ground elements while the total number of the finite elements are constant, the computational time is significantly reduced. Therefore study on changing accuracy versus ground element resolution may be an important factor in choosing ground element size. In addition to it the effective properties of arrangements of the strong and weak materials (black and white elements) in a checkerboard fashion are also investigated. The Michell-type problem is investigated by the minimization of the weight of the structure subjected to a compliance condition. It is shown that when four-node quadrilateral elements are involved and the size of the ground elements are varied, these constraints result in a numerically induced, artificially high stiffness and different optimal solution patterns. This can account for the formation of checkerboard patterns in continuous layout optimization problems of compliance minimization.

\section{Methodology}

Great number of design variables is a great challenge in optimization. Most of the methods are not able to solve that very demanding task. So there is a need for developing an algorithm to solve such a large topological optimization. This very important and popular topic was of interest to many scientists. Our approach is based on Karush-Kuhn-Tucker conditions described in detail in [10]. This SIMP (Solid Isotropic Material with Penalization) type algorithm is rather popular and has a long history $[3,5,19]$. It was found that the quality of the discretization has an influence on the optimal solution (on the optimal topology).

It was found in earlier studies (Rozvany [14]) that the quality control can be performed by mesh refinement. Contrary to the theoretical trends the "efficiency" decreased with refinement of the element in numerical experiments. The reason was that the number of finite elements and the number of ground elements were increased simultaneously, keeping the number of finite elements per ground element constant. The coarser net of larger size elements increased the discretization error, erroneously increasing the stiffness and decreasing the compliance (even without corner contacts in the solution). The above problem was overcome in the improved experiments (Rozvany [14]) in which the total number of the finite elements was kept constant in all calculations, but the number of ground elements was progressively increased (i.e. the number of FEs per ground element decreased).

In the following the ground element size and the variation of the Poisson's ratio is investigated in connection with the optimal topology. It is noted that the original Michell structures composed of members having uniaxial stress and generally zero Poisson's ratio is used in the numerical problems for the perforated plates.

\subsection{Problem formulation}

This section is based on our SIMP type algorithm [10]. Let us consider a plane stress or plane strain structure with rectangular element discretization using uniform rectangular mesh with elements $g=1, \ldots, G_{e}$. Due to the "checker board effect" described later (see Fig. 2) each element is usually subdivided to several sub-elements (Fig. 3). The structure is subjected to static load and boundary condition. The structure has also been imposed displacement constraints. The objective function is the weight of the structure which can be expressed as:

$$
W=\sum_{g=1}^{G_{e}} \gamma_{g} A_{g} t_{g}
$$

where $\gamma_{g}$ is the weight of the ground element, $A_{g}$ is area of the ground element, and $t_{g}$ is the thickness factor. The last one is also a design variable in topology optimization tasks. The factor takes values from the range $[0,1]$, however, we strive to have values of either $t_{\text {min }}=10^{-6}$ or $t_{\text {max }}=1$. It is caused by numerical reasons. An optimized structure should have only two states: either material is present or there is void. So optimization process tends to eliminate intermediate values of thickness. To achieve this effect thickness in equation (1) is penalized as follows:

$$
W=\sum_{g=1}^{G_{e}} \gamma_{g} A_{g} t_{g}^{\frac{1}{p}}
$$

Penalization minimizes value of the weight for limit values (0 or 1$)$.

The topology optimization problem can be formulated as follows:

- $\min W$,

- simple bounds: $t_{\text {min }} \leq t_{g} \leq t_{\text {max }}$,

- inequality constraint $u_{D} \leq \Delta_{D}$,

where $u_{D}$ is a chosen displacement in the structure and $\Delta_{D}$ is the prescribed permitted value of this displacement.

The inequality constraint can be also written in the form:

$$
\hat{\mathbf{u}}_{\mathbf{d}}^{\mathrm{T}} \mathbf{K u}-\Delta_{d} \leq 0 \quad(d=1, \ldots, D)
$$

where $\hat{\mathbf{u}}_{\mathbf{d}}^{\mathrm{T}}$ is the virtual displacement vector of virtual loads, $\mathbf{u}$ is the displacement caused by static load vector $\mathbf{P}, \Delta d$ is the prescribed displacement threshold. $\mathbf{K}$ is the structural linear stiffness matrix. As it was proven in [2] the inequality condition (3) can be rewritten as:

$$
\mathbf{u}^{\mathrm{T}} \mathbf{K u}-\mathrm{C} \leq 0
$$

where $\mathrm{C}$ is the compliance of the structure. In the case of static load compliance is a monotonic function of load intensity. The topology optimization problem is to minimize penalized weight (2) subjected to inequality constraint: 


$$
\begin{gathered}
\min W=\sum_{g=1}^{G_{e}} \gamma_{g} A_{g} t_{g}{ }^{\frac{1}{p}}, \\
\left\{\begin{array}{l}
\mathbf{u}^{\mathrm{T}} K u-C \leq 0, \\
t_{\text {min }}-t_{g} \leq 0, \\
t_{g}-t_{\text {max }} \leq 0 .
\end{array}\right.
\end{gathered}
$$

subject to

\subsection{Lagrange duality formulation}

Using formulation (5), Lagrange function can be written in the form as follows:

$$
\begin{gathered}
\mathcal{L}\left(t_{g}, v, \alpha_{g}, \beta_{g}, h_{1}, h_{2 g}, h_{3 g}\right)=\sum_{g=1}^{G_{e}} \gamma_{g} A_{g} t_{g}^{\frac{1}{p}}+v\left(\mathbf{u}^{\mathrm{T}} \mathbf{K u}-C+h_{1}^{2}\right)+ \\
+\sum_{g=1}^{G_{e}} \alpha_{g}\left(t_{\min }-t_{g}+h_{2 g}^{2}\right)+\sum_{g=1}^{G_{e}} \beta_{g}\left(t_{g}-t_{\max }+h_{3 g}^{2}\right),
\end{gathered}
$$

where $v, \alpha_{g}, \beta_{g}$ are the Lagrange multipliers and $h_{1}, h_{2 g}, h_{3 g}$ are slack variables. Using the standard numerical procedure the Karush-Kuhn-Tucker conditions can be written as follows.

\subsection{Karush-Kuhn-Tucker conditions}

Stationary conditions are based on derivatives with respect to Lagrange multipliers. Differentiating eq. (6) we obtain:

$$
\begin{gathered}
\frac{\partial \mathcal{L}}{\partial t_{g}}=\frac{1}{p} \gamma_{g} A_{g} t_{g}^{\frac{1-p}{p}}-v\left(\frac{\partial \mathbf{u}^{\mathrm{T}}}{\partial t_{g}} \mathbf{K u}+\mathbf{u}^{\mathrm{T}} \frac{\partial \mathbf{K}}{\partial t_{g}} \mathbf{u}+\mathbf{u}^{\mathrm{T}} \mathbf{K} \frac{\partial \mathbf{u}}{\partial t_{g}}\right)-\alpha_{g}+\boldsymbol{\beta}_{g}=0 \\
\left(g=1, \ldots, G_{e}\right)
\end{gathered}
$$

Due to symmetry of the stiffness matrix and linear relation between $\mathbf{K}$ and $t_{g}$ the above equation can be simplified in the form below:

$$
\frac{\partial \mathcal{L}}{\partial t_{g}}=\frac{1}{p} \gamma_{g} A_{g} t_{g}^{\frac{1-p}{p}}-v \frac{\mathrm{R}_{g}}{t_{g}{ }^{2}}-\alpha_{g}+\beta_{g}=0,
$$

where

$$
R_{g}=t_{g}^{2} \sum_{e=1}^{E_{g}} \mathbf{u}_{g e}^{T} \tilde{\mathrm{K}}_{g e} \mathrm{u}_{g e},
$$

and $\tilde{\mathrm{K}}_{g e}$ is the finite element stiffness matrix computed for $t_{g}=1$. Due to compactness rest of derivatives is presented in simplified form:

$$
\begin{gathered}
\frac{\partial \mathcal{L}}{\partial v}=\mathbf{u}^{\mathrm{T}} \mathbf{K u}-C+h_{1}^{2}=0 \text { and } \frac{\partial \mathcal{L}}{\partial h_{1}}=2 v h_{1}, \\
\frac{\partial \mathcal{L}}{\partial \alpha_{g}}=-t_{g}+t_{\text {min }}+h_{2 g}^{2}=0 \text { and } \frac{\partial \mathcal{L}}{\partial h_{2 g}}=2 \alpha_{g} h_{2 g}, \\
\frac{\partial \mathcal{L}}{\partial \beta_{g}}=t_{g}-t_{\text {max }}+h_{3 g}^{2}=0 \text { and } \frac{\partial \mathcal{L}}{\partial h_{3 g}}=2 \alpha_{g} h_{3 g} .
\end{gathered}
$$

\subsection{Iterative updating formulas}

Lagrange multipliers and slack variables can be computed iteratively. Thickness, which is also a design variable, is calculated by iterative formulas. There are three possible scenarios of updating a design variable:

First, when $t_{\min }<t_{g}<t_{\max }$, ground elements are called "active: $A \neq 0 ", \alpha_{g}=\beta_{g}=0$, updating formula can be derived form (8):

$$
t_{g}=\left(\frac{v p R_{g}}{A_{g} \gamma_{g}}\right)^{\frac{p}{p+1}}
$$

In the second case ("passive: $\boldsymbol{P} \neq 0$ ") $t_{g}=t_{\min }$, Lagrange multipliers are $\alpha_{g} \geq 0, h_{2 g}=0$, and from (8) one can obtain:

$$
t_{g} \geq\left(\frac{v p R_{g}}{A_{g} \gamma_{g}}\right)^{\frac{p}{p+1}}
$$

So if smaller value of $t_{g}$ than $t_{\text {min }}$ is calculated, equation (8) is satisfied by $t_{g}=t_{\min }$.

In the third case ("passive: $\boldsymbol{P} \neq 0$ "), where $t_{g}=t_{\text {max }}$, the corresponding Lagrange multipliers are $\beta_{g} \geq 0, h_{3 g}=0$ and (8) implies:

$$
t_{g} \leq\left(\frac{v p R_{g}}{A_{g} \gamma_{g}}\right)^{\frac{p}{p+1}}
$$

To avoid some numerical problems it is strongly recommended to set a minimum value to some small value, for example $t_{\min }=10^{-6}$ Now we derive the final iterative formula on which the topology optimization algorithm is based on. While compliance condition (5) is active this condition can be rewritten as:

$$
C-\sum_{g=1}^{G_{e}} \frac{\mathrm{R}_{g}}{t_{g}}=0 .
$$

Substituting into (16) relation (13) yields:

$$
C-\sum_{g \in P} \frac{\mathrm{R}_{g}}{t_{g}}=\sum_{g \in A} \frac{\mathrm{R}_{g}}{t_{g}}=\sum_{g \in A} \frac{\mathrm{R}_{g}}{\left(\frac{v p R_{g}}{A_{g} \gamma_{g}}\right)^{\frac{p}{p+1}}},
$$

rearranging with respect to $v$ :

$$
v^{\frac{p}{p+1}}=\frac{\sum_{g \in A}\left(\frac{A_{g} \gamma_{g}}{p}\right)^{\frac{p}{p+1}} R^{\frac{1}{p+1}}}{C-\sum_{g \in P} \frac{\mathrm{R}_{g}}{t_{g}}} \quad(\text { for } \mathcal{A} \neq 0) .
$$

To update $t_{g}$ Lagrange multiplier have to be computed from eq. (18) and new thickness from formula (13).

Iterative algorithm of topology optimization can be enumerated as follow:

1. Create FE space model together with ground elements and boundary conditions.

2. Assume simple bound values of design values $t_{g}, t_{\min }=10^{-6}$, $t_{\max }=1$.

3. Specify maximum of compliance $\mathrm{C}_{\max }=150 \% * \mathrm{C}$ - corresponding to $t_{g}=t_{\max }$ for all elements.

4. Initialize penalty parameter $p=1$. The parameter will evolve during optimization progress.

5. Solve FEM task

6. Compute element compliance $C_{e}=\mathbf{u}_{e}^{T} \tilde{\mathbf{K}}_{e} \mathbf{u}_{e}$, where $\mathbf{u}_{e}$ is current element displacement vector, and $R_{g}$ from equation (9).

7. Compute Lagrange multipliers. 
8. Calculate new element thicknesses from (13).

9. Update sets of active and passive elements according to:

\begin{tabular}{ccc}
\hline Value & If & Element set \\
\hline$t_{g, \text { new }}=t_{\min }$ & $t_{g, \text { new }} \leq t_{\min }=10^{-6}$ & $e \in \mathcal{P}$ \\
$t_{g, \text { new }}=t_{\max }$ & $t_{g, \text { new }} \geq t_{\max }=1$ & $e \in \mathcal{P}$ \\
$t_{g, \text { new }}=t_{g, \text { new }}$ & $t_{g, \text { new }} \leq t_{g, \text { new }}=10^{-6}$ & $e \in \mathcal{A}$ \\
\hline
\end{tabular}

10. If there are some changes in set of active and passive element go to 5 (FEM computations).

11. If there are no changes in passive and active sets change penalty parameter $p$ according to scheme:

\begin{tabular}{ccc}
\hline$p$ value range & Increment & Go to \\
\hline $1-1.45$ & 0.1 & 5 \\
$1.5-3$ & 0.15 & 5 \\
$3-15$ & 0.25 & 5 \\
$>15$ & - & 12 \\
\hline
\end{tabular}

\section{Stop.}

\subsection{Idea of ground element}

In plane stress state the thickness of finite element can be treated as the design variable. Unfortunately if each finite element thickness is treated as an independent design variable, it leads to unexpected effect called "checker board effect". This phenomenon is illustrated in Fig. 1. Enlarged part of topology solution is presented in Fig. 2 . Black and white elements are adjacent to each other alternately. This solution cannot be regarded as a correct one. To avoid this undesirable phenomenon several adjacent finite elements are assigned the same value of design variables (thickness). This group (Fig. 3) is called ground element.

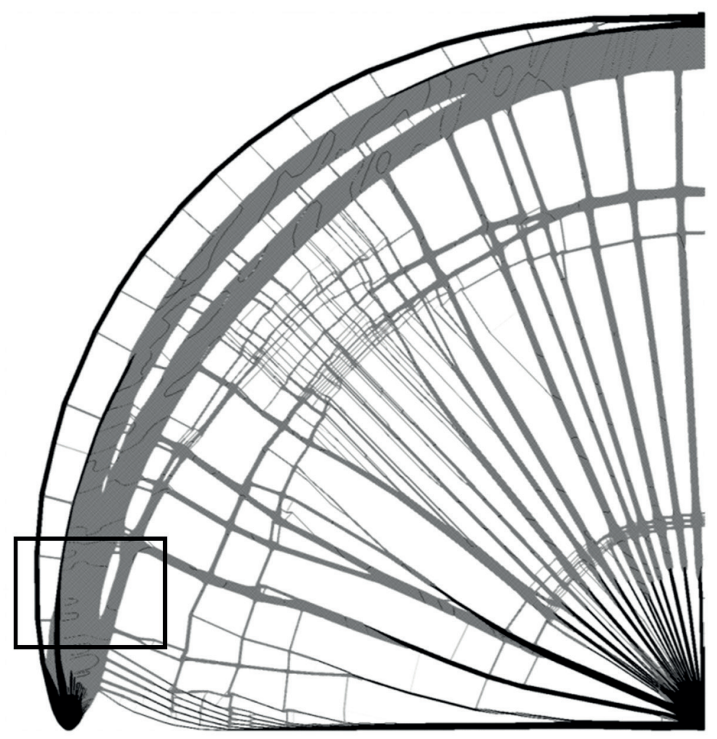

Fig. 1 Solution with ground element equal to finite element

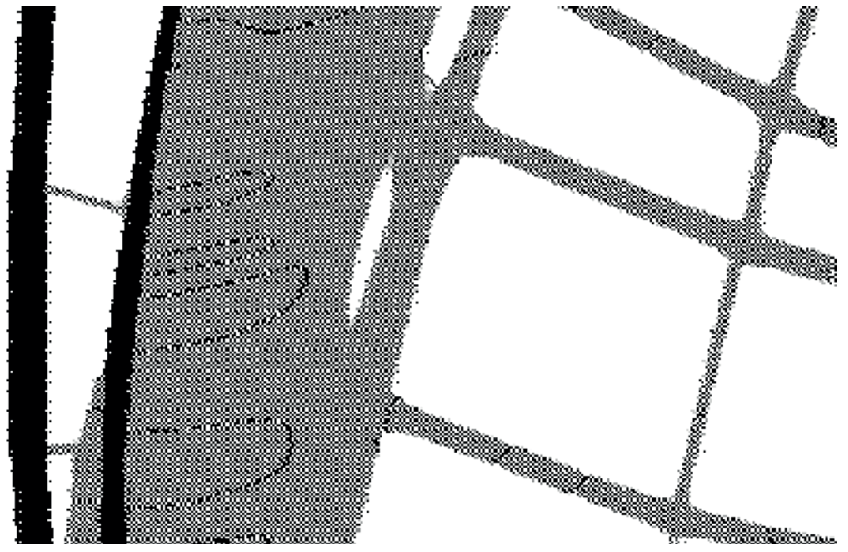

Fig. 2 Checker board effect with ground element size $=1$

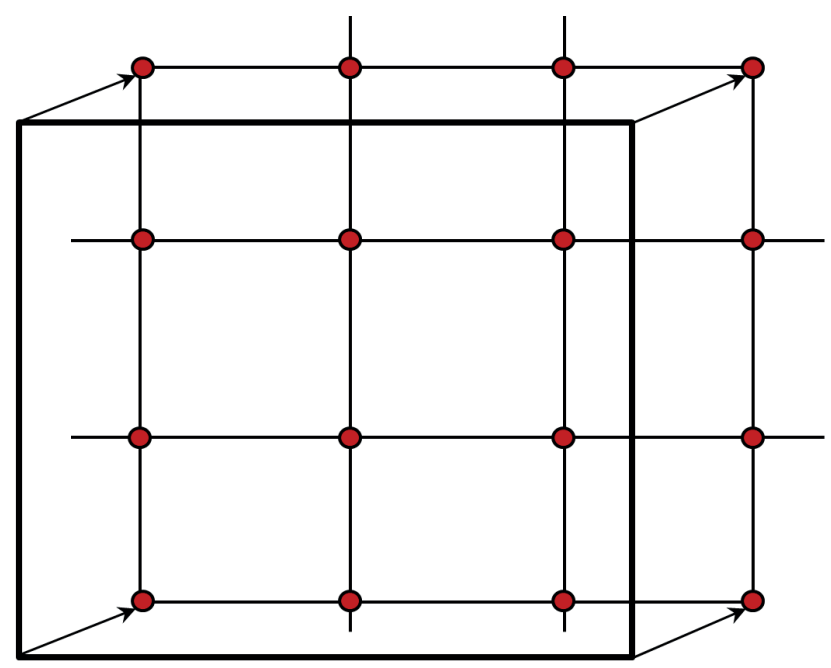

Fig. 3 Ground element idea $g e=3$

Usually choosing ground element of size two is enough to obtain proper results (Fig. 3). Greater size of the ground element causes faster topology optimization convergence but decreases resolution. In this paper we will check the influence of the ground element size on the results.

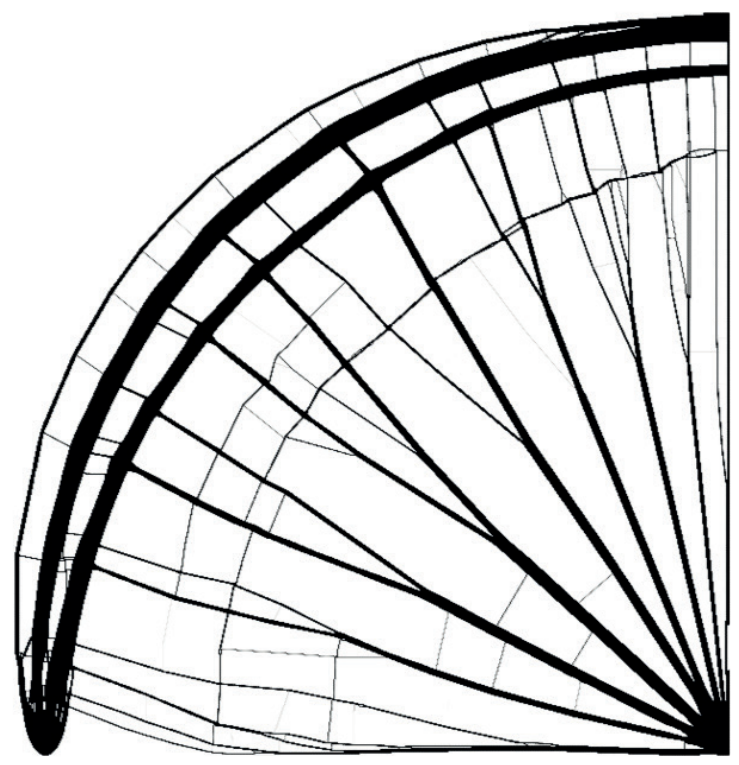

Fig. 4 Solution with ground element size $g e=2$. No checker board effect is observed. 


\section{Numerical examples}

\subsection{Example 1}

Several numerical examples have been investigated to illustrate the topological parametric study. The first example is shown in Figure 2. It is a plane stress beam with one force acting at the bottom of the beam in the middle distance between supports. We benefit on symmetry of the beam therefore a square mesh representing half of the beam will be taken into consideration. To achieve accurate results high resolution rectangular mesh will be used with dimension $1320 \times 1320$ elements. It makes around 3.5 million degrees of freedom.

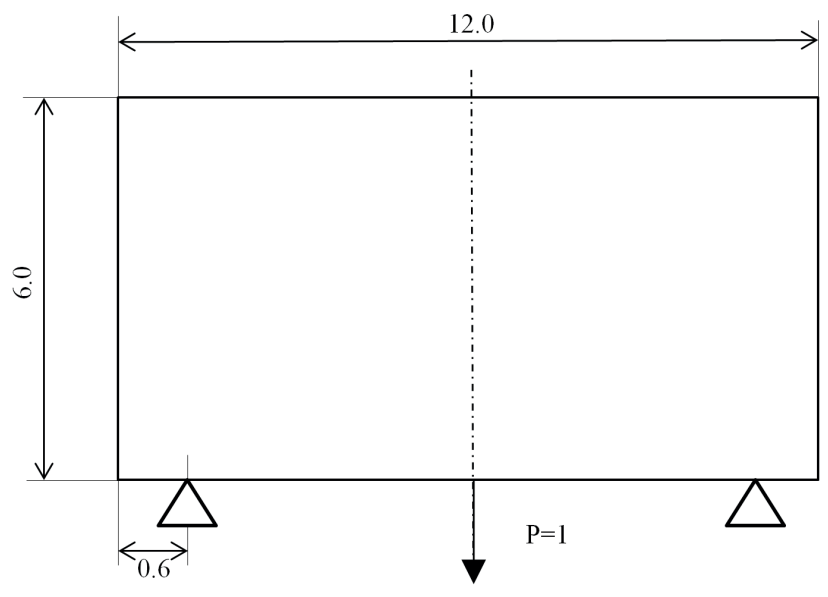

Fig. 5 Beam scheme

Topology optimization will be performed for several values of Poisson ratio $v=0,0.1,0.2,0.3,0.4$. We also would like to observe influence of ground element size on optimal shape. Therefore topology optimization will be performed for a set of ground element sizes: $g e=(1,2,3,4,5,6,8,10,12,24)$. It is worth mentioning that the Finite Element mesh dimension is always the same (described above) so there is no impact of ground element size on single finite analysis time. Detailed results are presented in Table 1. Plane stress material was isotropic with Young modulus $E=1$.

Computations were performed on HPC to benefit on sparse cluster architecture. In this very demanding task parallel computing plays a crucial role. HPC solution allows for even up to 10 topology optimization analysis performed simultaneously (It depends on the HPC server load). Another level of parallelism used in computations was the multiprocessor architecture of particular cluster node. Linear equation solver Pardiso subroutine implemented in multithreaded version was able to use this type of hardware significantly decreasing solution time.

Schemas of all solutions for each parameter combinations are presented in Table 1 .

\subsection{Example 2}

Three plane stress problems presented in Fig. 6 were computed. These schemas differ only in support mode. The optimal structures as well as the optimal volume parametric study are presented in Tables 2 to 4 and Fig. 7.

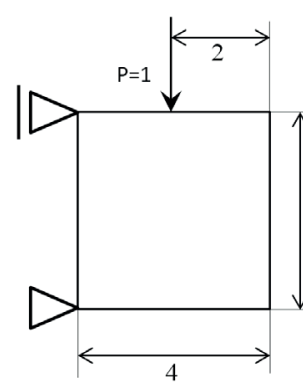

a)

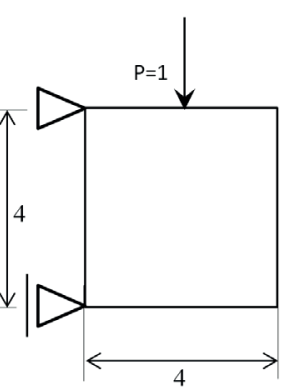

b)

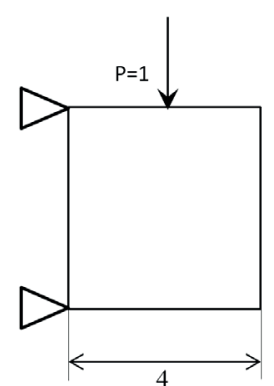

c)
Fig. 6 Three schemes differing in support mode.

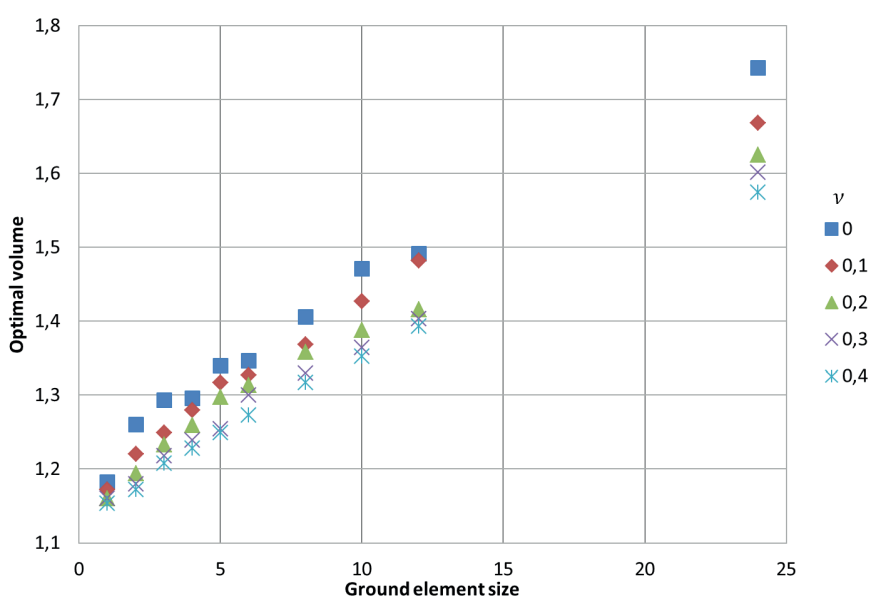

Fig. 7 Optimal volume for different ground element size and Poisson ratio.

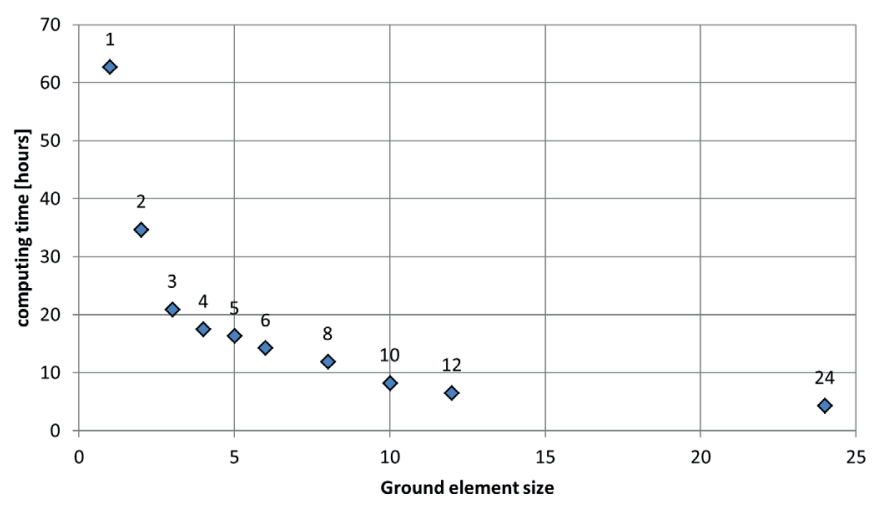

Fig. 8 Computational times vs ground element size. 


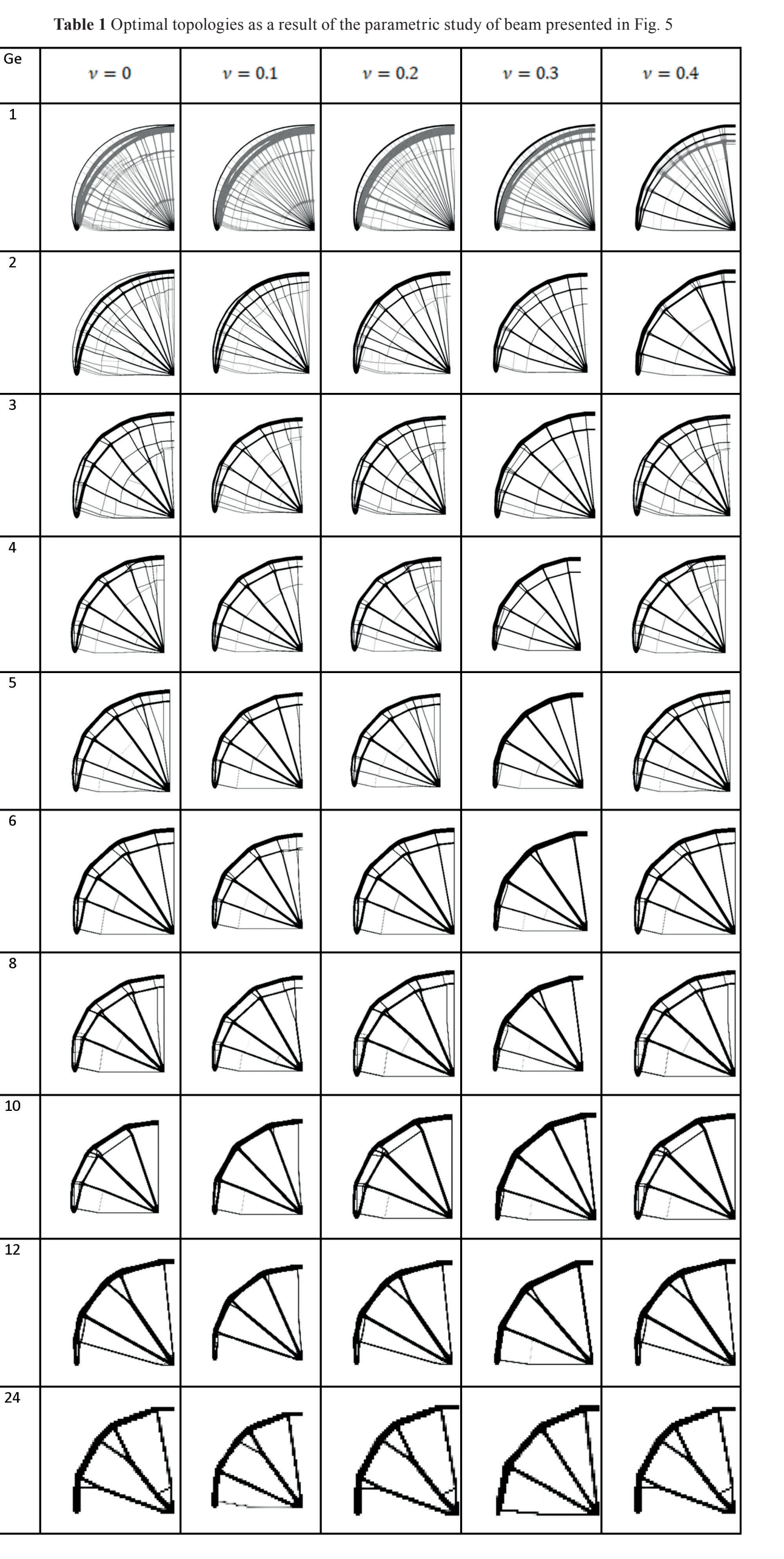


Table 2 Topology optimization results for Fig. $6 \mathrm{a}$

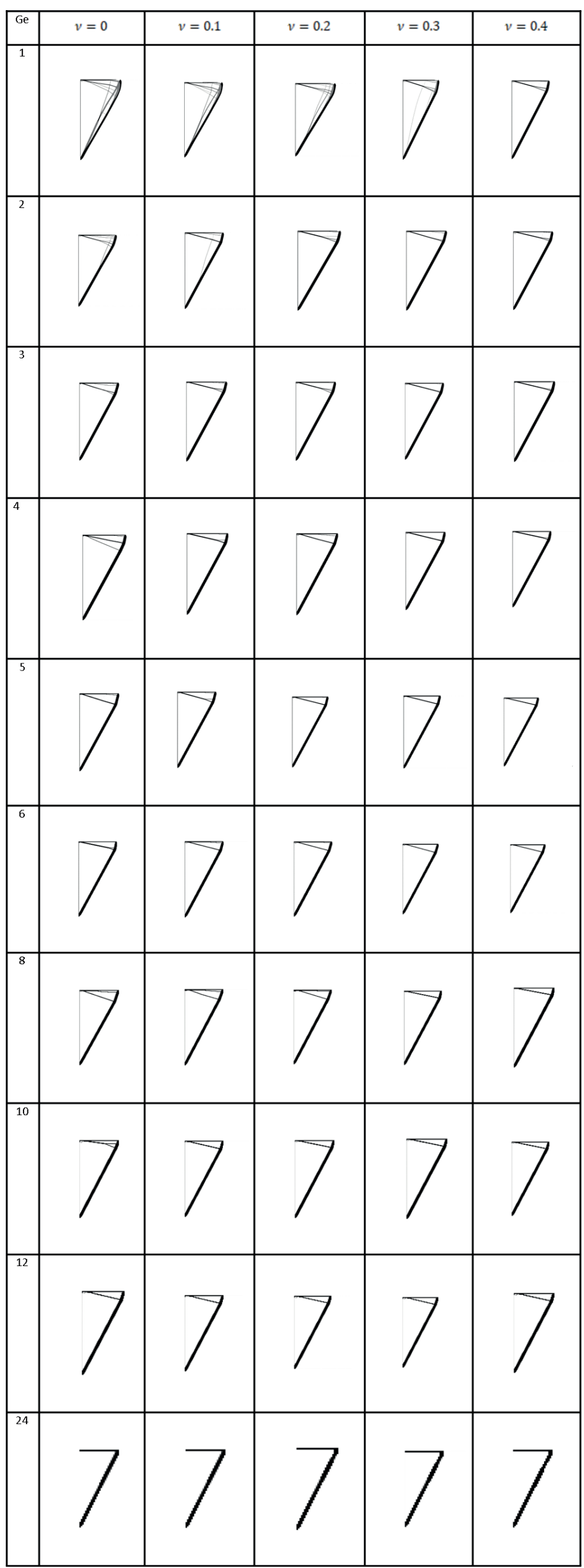


Table 3 Topology optimization results for Fig. $6 \mathrm{~b}$

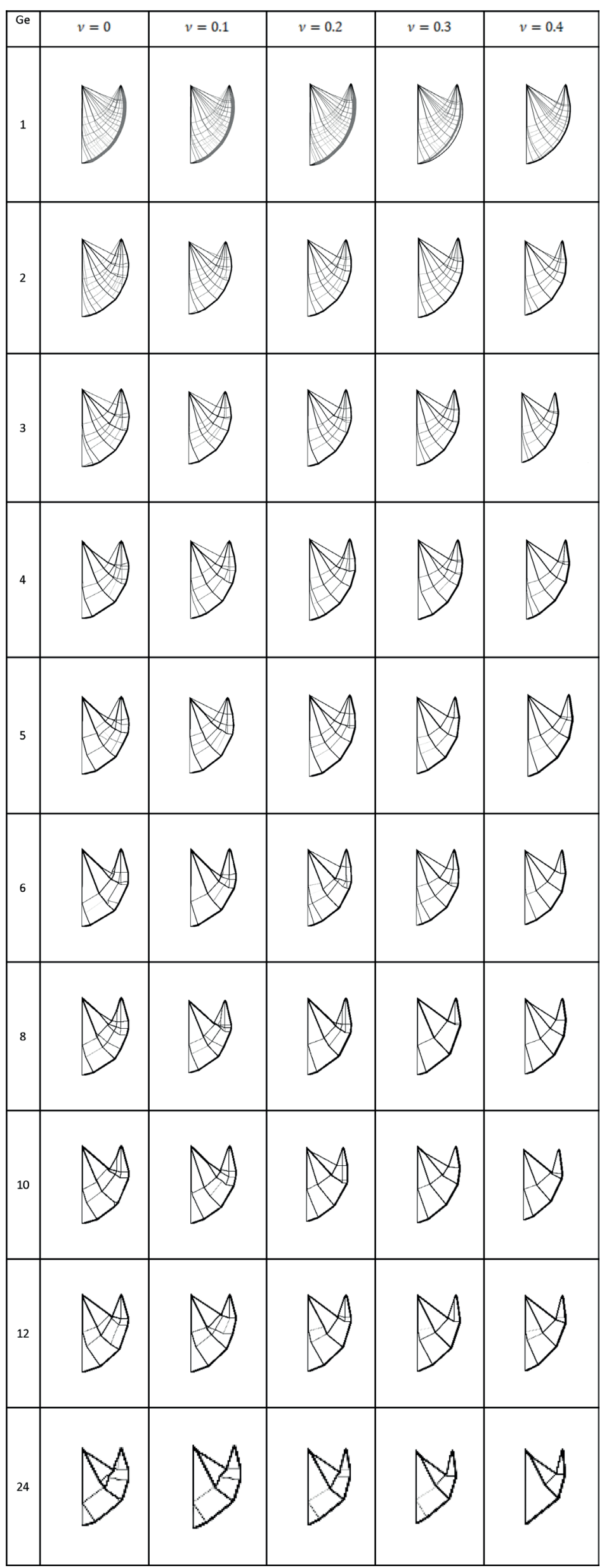


Table 4 Topology optimization results for Fig. $6 \mathrm{c}$

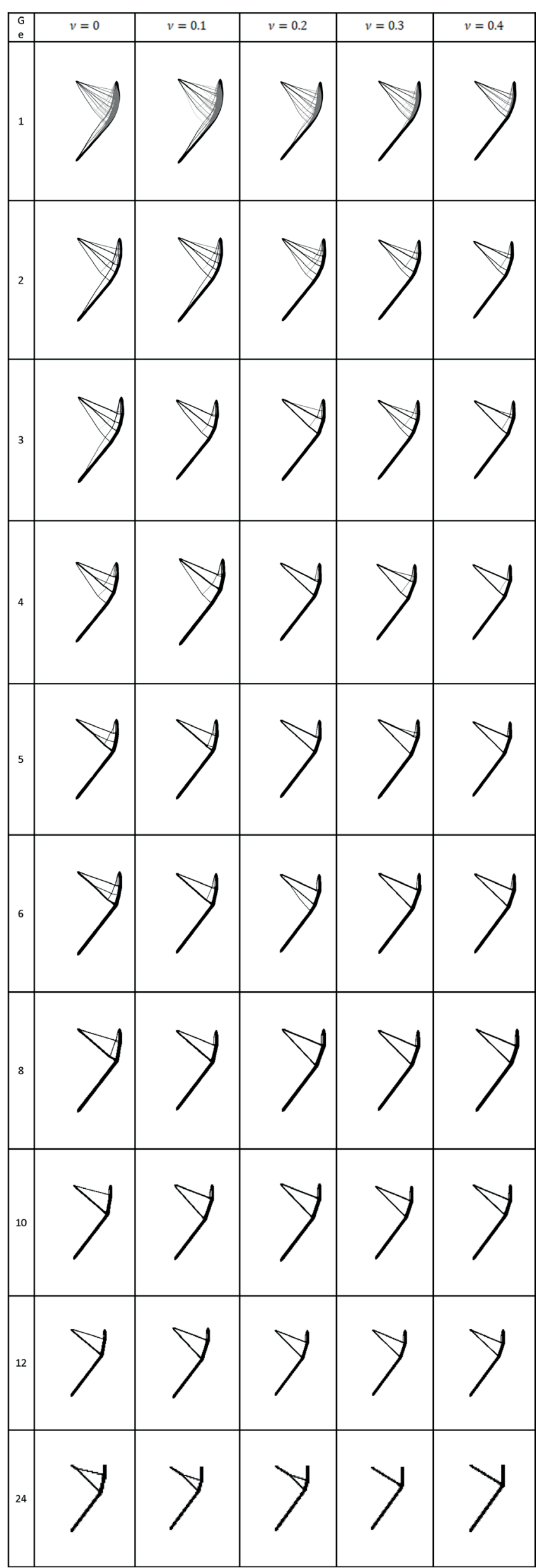




\section{Conclusions}

Computing time strongly depends on the size of ground elements (see Fig. 8) for the small sizes. Although we do not change the dimension of the finite element model, the calculation time varies considerably. Ground element size $E_{g}=2$ reduces computational time almost by half. Increasing it to $E_{g}=3$ we observe another significant time reduction (approximately half). But beyond that we find the trend change. From size $E_{g}=4$ to $E_{g}=24$ the speeding up of the calculations is linear and only a few percent. So ground element size of $E_{g}=3$ is the recommended choice from efficiency point of view.

\section{Acknowledgements}

The present study was supported by the National Research, Development and Innovation Office (grant K 119440) and ) and by the joint grant of the Hungarian and the Polish Academy of Sciences.

\section{References}

[1] Allaire, G., Kohn, R.V. "Topology optimization and optimal shape design using homogenization.”. In: Topology design of structures. NATO ASI Series (Series E: Applied Sciences), vol 227. (Bendsøe, M. P. \& Mota Soares, C. A. (Eds.)), pp. 207-218. Springer, Dordrecht. 1993. https://doi. org/10.1007/978-94-011-1804-0_14

[2] Beckers, M. "Topology optimization using a dual method with discrete variables". Structural Optimization, 17(1), pp. 14-24. 1999. https://doi. org/10.1007/BF01197709

[3] Bendsøe, M. P. "Optimization of structural topology, shape and material.”. Springer, Berlin, Heidelberg, New York. 1995. https://doi. org/10.1007/978-3-662-03115-5

[4] Bendøse, M. P., Kikuchi N. "Generating optimal topologies in optimal design using a homogenization method.". Computer Methods in Applied Mechanics and Engineering, 71(2), pp. 197-224. 1988. https://doi. org/10.1016/0045-7825(88)90086-2

[5] Bendsøe, M. P., Sigmund, O. "Topology optimization. Theory, methods and applications.”. Springer-Verlag. Berlin Heidelberg. 2003. https://doi. org/10.1007/978-3-662-05086-6

[6] Diaz, A., Sigmund, O. "Checkerboard patterns in layout optimization.”. Structural Optimization, 10(1), pp. 40-45. 1995. https://doi.org/10.1007/ BF01743693

[7] Hegemier, G. A., Prager, W. “On Michell trusses.”. International Journal of Mechanical Sciences, 11(2), pp. 209-215. 1969. https://doi.org/ 10.1016/0020-7403(69)90006-X

[8] Jog, C., Haber, R. "Stability of finite element models for distributed-parameter optimization and topology design.”. Computer Methods in Applied Mechanics and Engineering, 130(3-4), pp. 203-226. 1996. https:// doi.org/10.1016/0045-7825(95)00928-0

[9] Langelaar, M. "The use of convex uniform honeycomb tessellations in structural topology optimization.". In: Proceedings of the 7th world congress on structural and multidisciplinary optimization. Seoul, South Korea, 21-25 May, 2007.

[10] Lógó, J. "New Type of Optimal Topologies by Iterative Method.". Mechanics Based Design of Structures and Machines, 33(2), pp. 149-171. 2005. https://doi.org/10.1081/SME-200067035

[11] Lógó, J. "SIMP type topology optimization procedure considering uncertain load position.”. Periodica Polytechnica Civil Engineering, 56(2), pp. 213-220. 2012. https://doi.org/10.3311/pp.ci.2012-2.07
[12] Lyness, J. N., Monegato, G. "Quadrature rules for regions having regular hexagonal symmetry.”. SIAM Journal on Numerical Analysis, 14(2), pp. 283-295. 1977. https://doi.org/10.1137/0714018

[13] Rozvany, G. I. N., Pomezanski, V, Querin, O. M., Gaspar, Z., Logo, J. “Corner contact suppression in topology optimization.”. In: Engineering Design Optimization. Proceedings of the 5th ASMO UK/ISSMO Conference. (Querin, O. M., Sienz, J., Toropov, V. V., Goslong, P. (Eds)). Stratford-upon-Avon, United Kingdom, July 12-13, 2004, Leeds: University of Leeds, 2005. pp. 33-40.

[14] Rozvany, G. I. N., Lewinski, T., Querin, O. M., Lógó, J. “Quality control in topology optimization using analytically derived benchmarks.”. In: 11th AIAA/ISSMO Multidisciplinary Analysis and Optimization Conference. Portsouth, Virginia, USA, AIAA paper No.: AIAA 2006-6940. pp 484-494. 2006. https://doi.org/10.2514/6.2006-6940

[15] Rozvany, G. I. N. "A Critical Review of Established Methods of Structural Topology Optimization.”. Structural and Multidisciplinary Optimization, 37(3), pp. 217-237. 2009. https://doi.org/10.1007/s00158-0070217-0

[16] Sigmund, O., Petersson, J. "Numerical instabilities in topology optimization: A survey on procedures dealing with checkerboards, mesh-dependencies and local minima.”. Structural Optimization, 16(1), pp. 6875. 1998. https://doi.org/10.1007/BF01214002

[17] Stolpe, M., Svanberg, K. "An alternative interpolation scheme for minimum compliance topology optimization.". Structural and Multidisciplinary Optimization, 22(2), pp. 116-124. 2001. https://doi.org/10.1007/ s001580100129

[18] Talischi, C., Paulino, G. H., Le, C. H. "Honeycomb Wachspress finite elements for structural topology optimization.”. Structural and Multidisciplinary Optimization, 37(6), pp. 569-583. 2009. https://doi.org/10.1007/ s00158-008-0261-4

[19] Zhou, M., Rozvany, G. I. N. "The COC algorithm. Part II: Topological, geometry and generalized shape optimization.”. Computer Methods in Applied Mechanics and Engineering, 69(1-3), pp. 197-224. 1991. https:// doi.org/10.1016/0045-7825(91)90046-9

[20] Hunyadi, M., Hegedüs, I. "The sensitivity of the flutter derivatives and the flutter speed to the eccentricity of the cross section.". Periodica Polytechnica Civil Engineering, 56(2), pp. 167-173. 2012. https://doi. org/10.3311/pp.ci.2012-2.03 